\title{
Modelling and Simulation of Free Floating Pig for Different Pipeline Inclination Angles
}

\author{
Dereje Engida Woldemichael, Fakhruldin Mohd Hashim, Mark Ovinis, Wen Ching Lee and Muhammad Hazim bin Mohd \\ Halim
}

Universiti Teknologi Petronas, Mechanical Engineering Department, 32610 Bandar Seri Iskandar, Perak, Malaysia

\begin{abstract}
This paper presents a modelling and simulation of free floating pig to determine the flow parameters to avoid pig stalling in pigging operation. A free floating spherical shaped pig was design and equipped with necessary sensors to detect leak along the pipeline. The free floating pig does not have internal or external power supply to navigate through the pipeline. Instead, it is being driven by the flowing medium. In order to avoid stalling of the pig, it is essential to conduct simulation to determine the necessary flow parameters for different inclination angles. Accordingly, a pipeline section with inclination of $0^{\circ}, 15^{\circ}, 30^{\circ}, 45^{\circ}, 60^{\circ}, 75^{\circ}$, and $90^{\circ}$ were modelled and simulated using ANSYS FLUENT 15.0 with water and oil as working medium. For each case, the minimum velocity required to propel the free floating pig through the inclination were determined. In addition, the trajectory of the free floating pig has been visualized in the simulation.
\end{abstract}

\section{Introduction}

Pipelines are the most efficient and low cost fluid transportation (oil, gas, or water) means over a long distance. Pipeline operators conduct periodic inspections to find flaws and damage which might lead to leakage of the product to the environment. Early detection of leaks in pipelines is essential to reduce product loss, damage to the environment, and high clean-up costs. Therefore, it is necessary to develop a robust system into leak monitoring techniques and apply them in field. There are a variety of leak detection methods with varying degree of accuracy.

Pipeline Inspection Gauge commonly known as pig is a device that is inserted into a pipeline and propelled by the pressure of the product flow or driven by external power. Pig can be used to physically separate different fluids, clean the pipeline, collect data, or inspect the condition of the pipeline. In oil and gas industry pigs are mainly used for cleaning and inspection purposes.

Unlike conventional pigs, a free floating pig reported in this paper is a spherical shaped pig that does not occupy the whole space in the pipe and does not require its own dedicate source of energy to drive through the pipeline. It is being driven by the medium while floating without interrupting the normal operation. Thus, the movement of the pig is highly dependent on many aspects such as pressure, inclination angle, temperature and type of fluid. This study is aimed to identify flow parameters required for the pig to travel through the pipeline at different inclination angles using simulation model.
Free floating pigs can be used to inspect most pipelines including unpiggable pipelines having small radius bends, change in diameter across the pipeline, tee joints and branched lines [1]. Even in a piggable pipelines, operators use pigs as their last option due to the increased risk of "stalled" pig and disruption of the normal operation in addition to cost of locating and removing the pig. Free floating pigs can be used to address this issue since the pig diameter is smaller than the pipeline and spherical shape can easily move through small bends provided that the pipeline is clean.

A spherical shaped free floating pig was designed to conduct leak detection in pipelines. Unlike smartball [2], [3] which uses acoustic signal transmitted through the medium for leak detection, the free floating pig reported in this paper uses pressure signal and combination of other sensors to detect and locate leak.

If pig get stuck during pigging operation, it can seriously affect the normal operation and result in high intervention costs [4]. Therefore, detailed pigging simulation is very important to study the effect of different parameters before the pigs are deployed. Yu et al [5] conducted simulation study using OLGA software for deepwater flow lines before pigging operation to predict the minimum stable flow rate. Xu \& Gong [6] developed a simplified pigging model for predicting the pigging operation in gas condensate horizontal pipelines and compared with simulation result from OLGA software. Tolmasquim and Nieckele [7] developed simulation model to assist in the control and design of pig operations through pipelines and predict the process variables. Esmaeilzadeh et al [8] modelled and simulated 
the pig in gas and liquid pipelines to predict the pig position, pig velocity, upstream optimum flow rate, and time to reach its destination. A computational fluid dynamics simulation using FLUENT was used by Zhu et al [9] to study the leaked oil flows from damaged submarine pipelines. From the above literatures, it is evident that conducting simulation study is critical before filed trial.

In practical application, the mini pig has to travel through a pipeline having a number of bends and varying inclination angles depending on the topography of the location where the pipeline is installed. The modelling and simulation of the free floating pig will help us to visualize the flow pattern, determine minimum pressure and flow rate required for the pig to freely float and driven by the medium for different inclination angles. In this study, commercial software package ANSYS FLUENT 15.0 is used. Oil and water were used as products in the pipeline.

\section{Simulation model}

The fluid flow model is created using the k-epsilon model ANSYS Fluent. The input parameters are the fluid type, diameter, mass and density of the free floating mini pig, and the inlet velocity. The geometry of a cut out section of the pipeline is created followed by meshing in ANSYS. The simulation results were visualized and analysed to determine the minimum velocity required to propel the free floating pig through the inclination and visualize its trajectory. The pressure and velocity profile of the fluid along the pipeline will also be visualized.

Two types of fluids namely oil and water were used as the working medium for the simulation with the properties shown in Table 1. Both oil and water are assumed to be incompressible fluids with fully developed flow pattern. Thus, "Simple" scheme was selected with "Least Square Cell Based" gradient for simulation of single phase flow. Discrete Phase Model (DPM) is chosen to simulate the movement of the free floating Pig. DPM is a solution that is designed to track the trajectory of the particle in a fluid flowing motion. DPM uses Lagrangian reference frame as the main reference where the position and velocity of individual particle are tracked independently.

Table 1. Properties of fluids considered in the simulation.

\begin{tabular}{|c|c|c|}
\hline Medium & Density $\left[\mathbf{k g} / \mathbf{m}^{\mathbf{3}}\right]$ & Viscosity $[\mathbf{k g} / \mathbf{m}-\mathbf{s}]$ \\
\hline Water & 998.2 & 0.001003 \\
\hline Oil & 830 & 0.00332 \\
\hline
\end{tabular}

\subsection{Geometric Modeling of the Inclined Pipeline}

The For numerical simulation, we considered a cut out section of a pipeline where the pipeline experience change in elevation at varying angle connected with straight section at both ends. Seven inclination angles were considered namely: $0^{\circ}, 15^{\circ}, 30^{\circ}, 45^{\circ}, 60^{\circ}, 75^{\circ}$, and $90^{\circ}$. The pipe diameter is set to be 100 millimetres (4 inch) and the length of the pipe is 1 meter at each section of the pipe.
Once the geometries are created for each inclination angle, the geometric models were meshed. Two key factors that need to be considered during meshing are aspect ratio and element quality. Aspect ratio is defined as the ratio between the longest dimensions to the shortest dimension of the quadrilateral element in the mesh and element quality basically determine the quality of the mesh. Table 2 shows the results on the meshing of the geometry.

Table 2. Mesh parameters

\begin{tabular}{|c|c|c|c|c|}
\hline $\begin{array}{c}\text { Inclinatio } \\
\text { n Angle } \\
\text { [degree] }\end{array}$ & $\begin{array}{c}\text { Number } \\
\text { of nodes }\end{array}$ & $\begin{array}{c}\text { Number } \\
\text { of } \\
\text { elements }\end{array}$ & $\begin{array}{c}\text { Average } \\
\text { element } \\
\text { quality }\end{array}$ & $\begin{array}{c}\text { Aspect } \\
\text { ratio }\end{array}$ \\
\hline 0 & 123000 & 118558 & 0.6 & 3.5 \\
\hline 15 & 1420288 & 1450626 & 0.8 & 2.7 \\
\hline 30 & 159259 & 170384 & 0.6 & 3.6 \\
\hline 45 & 226334 & 242574 & 0.6 & 3.5 \\
\hline 60 & 433645 & 455227 & 0.7 & 3.4 \\
\hline 75 & 486707 & 490363 & 0.7 & 3.3 \\
\hline 90 & 2051650 & 1932039 & 0.7 & 2.9 \\
\hline
\end{tabular}

After the meshing has been done, the necessary parameters and fluid properties were set up in the system. The simulation was started with initial velocity of $0.5 \mathrm{~m} / \mathrm{s}$ and if the free floating pig was unable to propel through the inclination, the setup for the fluid velocity will be increased with an increment of $0.1 \mathrm{~m} / \mathrm{s}$. The steps will be repeated until the ideal initial velocity have been achieved.

\subsection{Modelling the free floating pig}

The free floating pig is modelled as a particle. The trajectories of the particle is computed in a Lagrangian frame using discrete phase models (DPM). The particle force balance equation given in equation 1 is integrated to get the particles trajectory.

$$
\frac{d u_{i}^{p}}{d t}=F_{D}\left(u_{i}-u_{p}\right)+\frac{g_{i}\left(\rho_{p}-\rho\right)}{\rho_{p}}+\frac{F_{i}}{\rho_{p}}
$$

where

$F_{D}\left(u_{i}-u_{p}\right)$ is drag force per unit particle mass

$\frac{g_{i}\left(\rho_{p}-\rho\right)}{\rho_{p}}$ is gravity force and

$\frac{F_{i}}{\rho_{p}}$ is additional forces: pressure gradient, Saffman lift, or other user defined force

In equation (1) $u_{i}$ is the fluid phase velocity, $u_{p}$ is the particle velocity, $\mu$ is the molecular viscosity of the fluid, $\rho$ is the fluid density, $\rho_{\mathrm{p}}$ is the density of the particle, and $D p$ is the particle diameter.

The forces acting on the free floating pig are gravitational, buoyance, drag, and lift forces. The gravitational and buoyance forces are constant. As depth increases, the pressure will increase. Therefore, buoyancy force is created due to the difference in pressure of the top side of the free floating pig and the bottom side; 
creating the upward thrust. In contrast, the drag and lift forces depend on the flow condition. Drag force is the force acting opposite to the direction of fluid flow due to the resistance acting on the free floating pig while the lift force is the force acting perpendicularly upward on the free floating pig relative to the motion of the fluid flow due to the pressure difference from opposite side of the free floating pig as a result of fluid flow past the pig. By increasing the velocity, the upward forces will be increased thus overcoming the downward force which will enable the free floating pig to propel through the inclination.

The simulation of the discrete phase trajectory in FLUENT assist us to determine the discrete phase inertia, hydrodynamic drag, and the force of gravity, for both steady and unsteady flows. Particle parameters have been set according to the parameters of the free floating pig such as the diameter of the pig, the density of the pig and flow rate of the transporting medium to simulate the situation as close as possible.

\section{Results and Discussion}

\subsection{Velocity profile and trajectory of the free floating pig}

The velocity profile for both fluids (oil and water) shows separation region at the bend for all inclination angles. This is because when fluid flows towards the pipe bends, there will be a radial force acting inward on the flow resulting in inertial force leading to increase velocity. Accordingly, a high velocity region is observed in the middle of the pipe and the region near the wall have a lower velocity due to friction for all inclination angles simulated. For both fluids, as the inclination angle increase, the separation angle also increase.

The trajectory of the free floating Pig at $30^{\circ}$ and $60^{\circ}$ inclination angles propelled by the flow of oil are shown in Fig. 1. In all cases the minimum fluid velocity to propel the free floating pig was determined and the trajectory of the free floating pig visualized. The average velocity of the free floating pig can also be determined from the simulation. For instance for the $30^{\circ}$ inclination shown in Fig. 1 (a), the average speed of the free floating pig ranges from $1.41 \mathrm{~m} / \mathrm{s}$ to $2.1 \mathrm{~m} / \mathrm{s}$, which is mainly in the green and yellow region of the velocity. Since the free floating pig is denser and heavier than the oil, the velocity of the free floating pig is slightly lower than the velocity of the transporting medium.

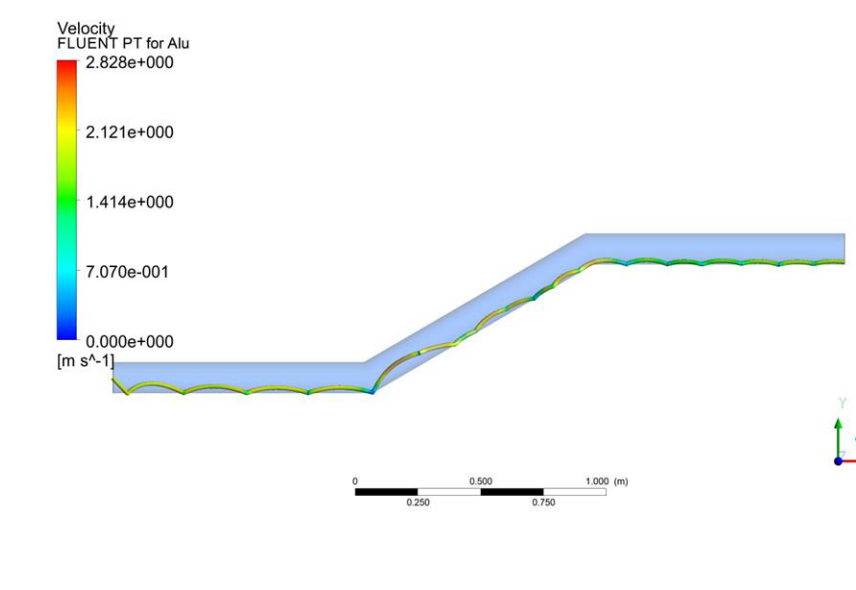

(a)

Figure 1. Free floating pig trajectory and velocity contour for (a) $30^{\circ}$ pipe geometry (b) $60^{\circ}$ pipe geometry

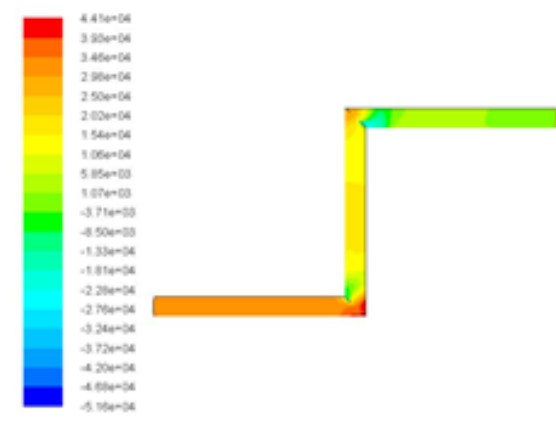

(a)

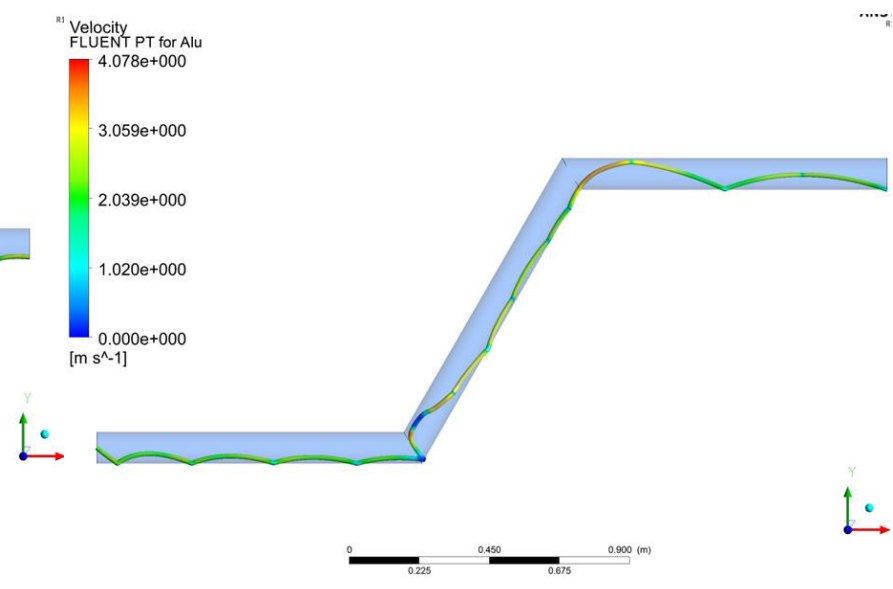

(b) 


\subsection{Pressure distribution}

The pressure profile in the pipeline changes from the inlet to the outlet as theoretically predicted with high pressure region at the bends for both fluids. This is due to the sudden change in direction leading to inertial effect and resistance from the pipe wall at the bend. Fig. 2 shows the pressure contour for $90^{\circ}$ pipe geometries with water and oil as working medium. Similar trends have been observed for other inclination angles. The higher the inclination angle, the higher pressure experienced at the bends.

\section{Conclusions}

The modeling and simulation of free floating pig for different inclination angle was presented in this paper. The simulation result assists in determining the critical flow parameters for the free floating pig to avoid stalling during operation. The simulation result assists us in visualizing the flow pattern, determine minimum pressure and flow rate required for the pig to freely float and driven by the medium for different inclination angles considered.

\section{Acknowledgement}

The authors would like to thank Universiti Teknologi PETRONAS, for the financial support in conducting this research under URIF grant.

\section{References}

1. Pikas, J. Beyond ECDA For Evaluating'Unpiggable'Pipelines: Advances In
Internal And External Tools. in Corrosion 2012. 2012. NACE International.

2. Kurtz, D., Developments in a Free-Swimming Acoustic Leak Detection System for Water Transmission Pipelines, in Pipelines 2006. p. 1-8, (2006).

3. Felipe Oliveira, et al., Smartball: $A$ new pipeline leak detection system, and its survey of two Petrobras/Transpetro pipeline field tests, in Rio Pipeline 2011 Conference \& Exposition. (2011).

4. Fung, G., et al. To pig or not to pig: the marlin experience with stuck pig. in Offshore Technology Conference. 2006. Offshore Technology Conference.

5. Yu, X., et al. Pigging Simulation Analysis of Deepwater Gas Tieback Flowlines in South China Sea. in The Twenty-third International Offshore and Polar Engineering Conference. International Society of Offshore and Polar Engineers. (2013).

6. Xu, X.-X. and J. Gong, Pigging simulation for horizontal gas-condensate pipelines with low-liquid loading. Journal of Petroleum Science and Engineering. 48(3-4): p. 272-280, (2005).

7. Tolmasquim, S.T. and A.O. Nieckele, Design and control of pig operations through pipelines. Journal of Petroleum Science and Engineering. 62(3-4): p. 102-110, (2008).

8. Esmaeilzadeh, F., D. Mowla, and M. Asemani, Mathematical modeling and simulation of pigging operation in gas and liquid pipelines. Journal of Petroleum Science and Engineering. 69(1-2): p. 100106, (2009).

9. Zhu, H., P. Lin, and Q. Pan, A CFD (computational fluid dynamic) simulation for oil leakage from damaged submarine pipeline. Energy. 64: p. 887-899, (2014). 